\title{
Questes
}

Revue pluridisciplinaire d'études médiévales

\section{Aveuglés par le Graal : les manifestations de la Providence dans le Lancelot Propre}

Isabelle Coumert

\section{(2) OpenEdition}

1 Journals

Édition électronique

URL : http://journals.openedition.org/questes/1036

DOI : 10.4000/questes. 1036

ISSN : 2109-9472

Éditeur

Les Amis de Questes

Édition imprimée

Date de publication : 15 septembre 2010

Pagination : 56-71

ISSN : 2102-7188

\section{Référence électronique}

Isabelle Coumert, «Aveuglés par le Graal : les manifestations de la Providence dans le Lancelot Propre

", Questes [En ligne], 19 | 2010, mis en ligne le 01 janvier 2014, consulté le 19 septembre 2020. URL : http://journals.openedition.org/questes/1036 ; DOI : https://doi.org/10.4000/questes.1036

\section{(c) Association des amis de «Questes »}




\section{Aveuglés par le Graal : les manifestations de la Providence dans le Lancelot Propre \\ Isabelle COUMERT}

Le Lancelot-Graal, grande somme romanesque anonyme du XIII ${ }^{\mathrm{e}}$ siècle, met en scène le monde arthurien depuis ses origines jusqu'à sa chute définitive après la bataille de Salesbières. Le cycle accorde une large place à la question religieuse, la Providence paraissant constamment veiller sur le destin du royaume de Logres. Évoqué tout au long du récit, depuis son transfert en Bretagne jusqu'à sa quête par la Table Ronde, le Graal constitue le symbole éclatant de cette omniprésence du sacré dans l'Histoire bretonne, au point d'éclipser d'autres manifestations moins évidentes de la divinité.

Nous nous intéresserons ici à la partie centrale du cycle, le Lancelot Propre, où le Graal paraît être mis le plus en retrait. Comment l'auteur entreprend-il de suggérer l'existence d'un projet divin par-delà les volontés individuelles de ses héros? Comment rendre manifeste l'action de la Providence en dépit des problèmes de mimesis que peut poser la représentation d'un mystère que seule la foi devrait rendre évident?

Nous nous pencherons tout d'abord sur la place que le Lancelot Propre accorde au Graal, avant d'évoquer le cas particulier de la Préparation à la Queste et les manifestations plus indirectes des intentions providentielles.

Le Lancelot Propre est l'élément central et de loin le plus développé du grand cycle du Lancelot-Graal. Pour préciser les enjeux de la question, il faudra commencer par replacer ce volume dans la continuité du cycle. Le 
Lancelot-Graal peut se décomposer en cinq éléments qui sont tous susceptibles de fonctionner en autonomie. Le premier livre du cycle est L'Estoire del Saint Graal, qui raconte la translatio du Graal depuis la Judée jusqu'en Bretagne, sous la garde de Joseph d'Arimathie et de ses descendants ${ }^{1}$. On ne peut manquer de remarquer que le modèle d'écriture de ce livre est biblique; il s'agit de retracer l'histoire d'une famille de justes et de saints luttant pour la foi, sur le modèle des Maccabées². Le second livre est L'Histoire de Merlin, qui raconte la naissance et la jeunesse de Merlin, ainsi que l'accession d'Arthur au pouvoir; c'est un livre inspiré du Merlin en vers de Robert de Boron, aujourd'hui perdu. Ce dernier a probablement été remanié pour pouvoir s'intégrer au cycle du Lancelot-Graal, mais le récit conserve l'inspiration mystique propre au cycle du Graal rédigé par Robert de Boron ${ }^{3}$ : Merlin y est présenté comme un véritable prophète chargé, outre ses prédictions, de jouer le rôle de guide auprès du jeune Arthur. Le livre central du cycle - celui qui nous intéresse ici - est le Lancelot propre, qui met en scène la jeunesse de Lancelot et les aventures de la Table Ronde. Il est généralement associé aux deux derniers volumes, la Queste del Saint Graal et la Mort le roi Artu, sous le nom de Lancelot en prose. La Queste del Saint Graal raconte la recherche puis la découverte du Graal par Galaad, Perceval et Bohort, et la Mort le roi Artu

1 Pour une analyse du merveilleux chrétien dans ce récit, voir Marie-Christine BRACCHIONI-GIORDANO, «Le merveilleux chrétien dans les récits apocryphes chrétiens et dans L'Estoire del Saint Graal : éléments de comparaison » et Gérard Gros, "L'uis de l'arche, ou merveilleux et théophanie dans L'Estoire del Saint Graal », in Francis GINGRAS, Françoise LAURENT, Frédérique LE NAN et Jean-René VALETTE (dir.), Furent les merveilles pruvees et les aventures truvees ». Hommage à Francis Dubost, Paris, Honoré Champion, 2005, p. 83-100 et p. 307-320 .

${ }^{2} \mathrm{Au} \mathrm{II}{ }^{\mathrm{e}}$ siècle avant $\mathrm{J}$.-C., les cinq frères Maccabées menèrent l'insurrection de la Palestine contre l'occupant syrien et fondèrent ainsi une dynastie de gouvernants. Le récit de cette période est rapporté par l'Ancien Testament dans le Premier livre des Maccabées. Le Second Livre évoque à son tour les persécutions, les martyres, et les campagnes menées par Judas Maccabée contre Antiochus.

${ }^{3}$ Rédigé dans la seconde moitié du XII ${ }^{\mathrm{e}}$ siècle, ce cycle comportait un Joseph d'Arimathie, pendant de L'Estoire del Graal, un Merlin, et un Perceval. 
la chute de l'univers arthurien, suite notamment à l'opposition entre la maison d'Arthur et celle de Lancelot, mais aussi au soulèvement de Mordret contre son père.

La question de l'unité de composition du cycle, qui a été longuement débattue $^{4}$, reste ouverte, compte tenu de ses dimensions et de ses variations de tonalité qui créent une impression persistante d'hétérogénéité. En effet, une lecture distanciée remarque une omniprésence du merveilleux chrétien dans L'Estoire del Saint Graal et dans la Queste del Saint Graal et une présence un peu plus affaiblie dans le Merlin et la Mort le roi Artu, alors que ce même merveilleux chrétien tiendrait une place beaucoup moins évidente dans le Lancelot propre, dominé par la figure de Lancelot et son destin marginal. Albert Pauphilet remarque ainsi que L'Estoire del Saint Graal et le Merlin « orientent le lecteur vers la légende du Vase Sacré comme vers le sujet essentiel du livre», alors que le Lancelot serait « un pur roman d'amour » précédant une Queste del Saint Graal « tout inspirée d'esprit religieux, dominée par l'idée, incarnée en Galaad, de la parfaite sainteté $»^{5}$. Le Lancelot propre n'aborde en effet que dans sa dernière partie, dite de Préparation à la Queste, la question du Graal : le prophète Merlin n'y apparaît plus que dans un court sommaire inaugural racontant comment il a été enfermé par Morgane dans une prison d'air. Ce récit de la disparition de Merlin au début du Lancelot en prose est du reste expédié en deux pages, probablement interpolées pour assurer la transition avec

\footnotetext{
${ }^{4}$ La question a été notamment débattue entre Ferdinand Lot, Albert Pauphilet et Jean Frappier. Voir Jean FRAPPIER, «Plaidoyer pour 1'“architecte”, contre une opinion d'Albert Pauphilet sur le Lancelot en prose », Romance Philology, VIII (1954-1955), p. 27-33. Il y entreprend de réfuter les propos d'Albert Pauphilet selon lequel «les parties de ce vaste ensemble sont bien, en général, faites ou refaites les unes pour les autres, et non rassemblées au hasard par de simples copistes » et " il n'était peut-être pas nécessaire de dépasser cette conception de l'unité de l'œuvre. »

${ }_{5}^{5}$ Albert Pauphilet, Le Legs du Moyen Âge, Melun, 1950, p. 212-217, cité par Jean FRAPPIER, «Plaidoyer pour 1'“architecte", contre une opinion d'Albert Pauphilet sur le Lancelot en prose », art. cit., p. 28.
} 
L'Histoire de Merlin ${ }^{6}$. Ainsi l'ouverture du Lancelot Propre, en recentrant aussitôt la narration sur la figure du jeune Lancelot, évince-t-elle avec Merlin et le Graal les figures centrales des deux volumes précédents, et, par là, l'enjeu mystique du cycle.

Avec l'effacement du Graal et de son prophète, le Lancelot Propre semble mettre longuement au second plan ce qui, dans les deux premiers volumes du cycle, occupait pourtant le devant de la narration. Le début du Lancelot propre paraît en effet s'opposer aux deux livres antérieurs, L'Estoire del Saint Graal et le Merlin. Désormais, plus de Graal, plus de guide prophétique : à l'ouverture du Lancelot, la question de la quête spirituelle semble provisoirement mise entre parenthèses. Il n'y aura pas non plus avant longtemps de ces interventions divines spectaculaires semblables à l'élection d'Arthur par retrait de l'épée, d'un côté (épisode de la fin du $\operatorname{Merlin}^{7}$ ), ou à l'apparition du Graal devant la cour, de l'autre (épisode qui ouvre la Queste del Saint Graal $^{8}$ ). Le Lancelot propre se centre aussitôt sur la figure de Lancelot, son enfance, ses premiers exploits, ses amours avec Guenièvre, mais bientôt il s'intéresse aussi aux quêtes menées par les autres chevaliers les plus en vue de la cour arthurienne : Bohort, Gauvain, Sagremor, Yvain, Keu, Girflet, Hector, Agravain - et la liste n'est pas close. Ces chevaliers passent l'essentiel de leur temps à lutter

\footnotetext{
${ }^{6}$ T. VII, VIa, 1-10, p. 38-43. Toutes les références au Lancelot en Prose renvoient aux neuf volumes de l'édition d'Alexandre Micha: Lancelot, Roman en prose $d u X^{X I I I}{ }^{e}$ siècle, Genève, Droz, 1978-1982.

${ }^{7}$ L'attribution à Dieu de ce miracle est réaffirmée tout au long de la cérémonie conduite par l'archevêque : "Artus, aller querre l'espee et la jostise dont vos devez desfandre Sainte Eglise et la crestianté sauver an toutes manieres a vostre pooir. », ROBERT DE Boron, Merlin, roman du XIII siècle, édition critique par Alexandre Micha, Genève, Droz, 1979, p. 289.

${ }^{8}$ L'apparition du Graal, dont le porteur reste invisible, s'accompagne de divers autres phénomènes surnaturels pour la plupart déjà constatés dans le Lancelot Propre: tonnerre, lumière aveuglante, mutisme forcé, odeurs d'épices, apparition de nourriture devant les convives. Arthur remercie aussitôt Notre-Seigneur de lui avoir accordé cette grâce, La Queste del Saint Graal, Roman du XIII siècle, publié par Albert PAUPHILET, Paris, Honoré Champion, «C.F.M.A. » n³3, 2003, p. 15-16.
} 
contre de mauvaises coutumes, à se chercher les uns les autres, à combattre et à se faire capturer ou délivrer. Les divers acteurs et les quêtes entrelacées sont si nombreux que le fil directeur du roman semble s'y perdre. L'épiphanie du Graal qui ouvre La Queste del Saint Graal et qui survient en cour plénière lors d'une fête de Pentecôte marque une rupture forte avec le relatif éparpillement des récits précédents, qui baignaient dans un univers plus léger, essentiellement chevaleresque et courtois.

Le Graal était pourtant perçu par les premiers lecteurs, sinon par le ou les auteurs originels, comme le véritable fil directeur de l'ensemble du cycle. C'est ce que montre la lecture du manuscrit de Modène; quelquefois considéré comme la véritable fin du Merlin de Robert de Boron, son récit d'une dernière visite de Merlin à la cour d'Arthur est sans doute une interpolation, là encore destinée à permettre la transition entre L'Histoire de Merlin et le Perceval dans le petit cycle du $\mathrm{Graal}^{9}$. Elle permet néanmoins de voir comment les médiévaux en comprenaient l'unité :

- Artu, vos estes rois, la Diu merci, et Uter vostre pere molt preudom; et fu faite la Table Reonde a son tans, qui fu faite en senefiance de celi u Nostre Sire sist au juesdi quant il dist que Judas le traïroit; et si fu faite encontre la table Joseph, qui estoree fu de par le Graal, quant il departi les buens des mauvais. Si vuel que vos saciés que il a eu deus rois en Bretagne devant vous qui ont esté roi de France et empereor de Rome, et je vuel bien que vous saciés que en Bretagne sera encore li tiers rois qui rois et emperere en sera et le conquerra a force sor les Romains. Et je vous di, si com jou ai le pooir de savoir les coses qui sont a avenir, que je les tieng de Nostre Segnor, que deus cens ans devant çou que vos fussiés nés, si vos fu ele prophétisie et jetés li sors sor vos. Mais il vous couvient ançois que vos soiés si preus et si vaillans que li Table Reonde soit rensaucie par vous. Et bien vos faç seur que vos ja emperere n'en serés desci adonc que li Table Reonde ert si essaucie que je vos dirai. [...]

(suit un résumé du Roman de l'Estoire dou Graal)

${ }^{9}$ On distingue le cycle court du Graal, constitué par les trois romans de Robert de Boron, du cycle long anonyme du Lancelot-Graal. 
Et li rois Peschiere si converse en ces illes d'Irlande en un des plus biaus lius del monde : et sacés que il est a greignor mesaise que onques fust hom et est cheus en grant maladie. Mais tant vous puis je bien dire que por viellece que il ait ne por enfermeté ne puet morir desci adont que uns cevaliers qui serra a la Table Reonde ait tant fait d'armes et de cevalerie, en tornois et par querre aventures, que il sera alosés del monde. Et cil, quant il s'ara si essaucié que il pora venir a la cort le rice Roi Pescheor et que li ara demandé de quoi li Graaus a servi et de quoi il sert, et tant tost sera garis. Et lors li acontera les secrees paroles de Nostre Segneur; si trespassera de vie a mort; et cil cevaliers ara le sanc Jhesu Crist en garde. Et lors charront li encantement par la terre de Bretagne et adont si sera la prophesie toute paracomplie. $^{10}$

Il s'agit d'une dernière apparition de Merlin à la cour d'Arthur, peu de temps après l'accession de ce dernier au pouvoir. Après avoir révélé à tous qu'Arthur est le fils d'Uter, il donne quelques recommandations au jeune roi, jouant une dernière fois le rôle de guide spirituel auprès de ce dernier. Se présentant comme un prophète inspiré, Merlin lui confie deux missions nouvelles, qui découlent toutes deux de son tout nouveau statut de souverain. La première concerne la Table Ronde; si cette dernière aurait été créée par Uter, il est du devoir d'Arthur de lui assurer prestige et rayonnement. Le sage esquisse alors un intéressant parallèle entre la Table Ronde, celle de la Cène, et celle de Joseph d'Arimathie. La fraternité chevaleresque de la Table Ronde perd ainsi son caractère profane pour s'inscrire dans le temps cyclique et sacré, de type ternaire, de la réactualisation périodique de la Table de la Cène. C'est ce qui explique la présence à chacune de ces tables d'un siège vide qui correspond au siège abandonné par Judas lors de sa trahison. L'hypocrite Moïse, qui avait voulu s'y asseoir près de Joseph d'Arimathie, y meurt immédiatement, de même que Brumant l'Orgueilleux en s'asseyant sur le Siège Périlleux à la toute

\footnotetext{
${ }^{10}$ Fin possible du Merlin (d'après le manuscrit de Modène), ROBERT DE BORON, Merlin, roman du XIII siècle, éd. cit., p. 293-297.
} 
fin du Lancelot Propre ${ }^{11}$. La deuxième mission confiée à Arthur découle de la première : il doit attirer à sa cour un chevalier suffisamment vaillant et sage pour accomplir la quête du Graal et mettre fin aux enchantements. Ces deux indications de Merlin, placées en conclusion du récit, donnent ainsi une grille de lecture pour la suite : dans l'idée de l'interpolateur, cette suite était le Perceval $^{12}$, mais cette interprétation pourrait aussi valoir pour le Lancelot Propre, selon le cycle dans lequel on se situe. Ainsi, si l'on suit ce guide d'interprétation, toutes les péripéties du Lancelot Propre ne font que narrer comment Arthur parvient à donner à sa cour un rayonnement suffisant pour attirer le meilleur chevalier du monde, qui dans un premier temps semble devoir être Lancelot, et qui sera en réalité son fils, Galaad. Le recul du merveilleux chrétien dans cette portion du cycle s'expliquerait alors par le fait que nous sommes dans un temps d'attente, de formation. Le Lancelot Propre fait avancer à sa manière l'histoire du Graal. L'histoire de la jeunesse de Lancelot est celle d'une élection - Lancelot est clairement qualifié comme le meilleur chevalier du monde et tenu pour tel par tous mais aussi d'une chute; à cause de son amour adultère pour Guenièvre, Lancelot ne pourra pas être le découvreur du Graal. S'il est écarté de la quête, il sera le père de Galaad, c'est-à-dire le père de l'Élu. Dans la dernière partie du Lancelot propre, le roman prend le temps de décrire la conception de Galaad et sa jeunesse, préparant ainsi la Queste. Le récit nous montre aussi les défaillances d'Arthur, vis-à-vis de Lancelot comme vis-à-vis de sa femme ${ }^{13}$; plusieurs fois adultère, le roi conçoit Mordret avec sa propre demi-sœur, préparant ainsi sa propre chute. Le lecteur

\footnotetext{
${ }^{11}$ T. VI, C, 38-39.

${ }^{12}$ Dans le manuscrit de Modène, c'est le Perceval qui suit le Merlin; c'est pourquoi Merlin annonce que celui qui découvrira le Graal en deviendra le Gardien : cela est vrai dans le Perceval, mais pas dans la Queste del Saint Graal.

${ }^{13}$ Arthur a ses propres torts envers Guenièvre, par exemple dans l'épisode de « la fausse Guenièvre », où il la répudie au profit d'une aventurière qui se fait passer pour elle, ou dans l'épisode de la Roche aux Saisnes, où il la trompe avec l'enchanteresse Camille (T.VIII, LXXIa).
} 
comprend alors que la découverte du Graal, sommet de sa gloire, ne sauvera pourtant pas le royaume arthurien.

Il faudra du reste nuancer les premières impressions de lecture en rappelant combien le Lancelot Propre, tout au long de sa seconde moitié, prend soin de préparer la transition avec le volume suivant. En dépit des nombreux épisodes intercalés, l'Agravain prépare la Queste del Saint Graal en montrant les visites successives de trois des chevaliers arthuriens à Corbenic $^{14}$ : Gauvain, Bohort et Lancelot visiteront chacun leur tour le château du Graal, avec plus ou moins de succès. Gauvain est le tout premier à pénétrer dans le château de Corbenic.

Endementiers qu'ils parloient issi, se regarde mesire Gauvain et voit par mi une verriere un blanc colon qui aportoit en sa boche .I. encenser d'or trop raemplis de totes les bones odors que cuers mortels porroit penser ne boche dire. Et lors furent tuit si amui que onques n'en i ot .I. sol qui mot deist, ains s'agenoillerent tuit, si tost com il virent le colon; et il s'en ala tot droit en une chambre. (...) De ceste aventure se merveille molt mesire Gauvain; si s'asiet avec les autres et voit qu'il sont tuit en proieres et en oroisons. (...) Ele issi de la chambre et porta entre ses .II. mains le plus riche vaissel qui onques par home terrien fust veus, et fu fes en samblance de calice, et le tint plus haut de son chief si que totes voies l'enclinoit. (...) Et ensi com la damoisele passe par devant le dois, si s'agenouille chescuns devant le saint vaissel et tantost sont les tables replenies de tos les bials mengiers que l'en porroit deviser; et li palés fu raemplis de si bones odors com se totes les espieces terrienes i fuissent espandues. $^{15}$

Il assiste au cortège du Graal, mais, seul des convives, voit son assiette rester vide. Un nain vient ensuite le menacer de son bâton pour l'expulser, puis le chevalier est grièvement blessé sur le Lit Périlleux et assiste au combat du serpent et du léopard. Il quitte le Palais Aventureux lié sur une

\footnotetext{
${ }^{14}$ Par ailleurs, Hector et Perceval, sans avoir accès à Corbenic, sont néanmoins guéris par le Graal (T.VI, CVI, 43-44).

${ }^{15}$ T.II, LXVI, 11-32. C'est nous qui soulignons.
} 
charrette, pendant que son écu est traîné dans la boue. Lancelot, pour sa part, séjourne par deux fois à Corbenic ; c'est lors de son premier séjour que Galaad est conçu. Longtemps après, à son retour de la campagne de Gaule, alors que le bannissement de la reine l'a frappé de folie, la vision du Graal lui rend la raison ${ }^{16}$. Bohort visite lui aussi deux fois Corbenic, il assiste une première fois au cortège ; la seconde fois, il rencontre Galaad avant d'assister à nouveau au cortège du Graal. Au terme de ses aventures dans le Palais Aventureux, il le verra enfin à découvert ${ }^{17}$.

Le Graal lors de ses différentes épiphanies présente des caractéristiques constantes : annoncé par une lumière éclatante, une odeur d'épices, une colombe portant un encensoir ou par le fracas du tonnerre, il est recouvert d'un voile blanc et ordinairement porté par une jeune fille. Il est doté du pouvoir de guérison et du pouvoir nourricier d'une corne d'abondance dans la mesure où il remplit les plats sur son passage. Son exposition apparemment quotidienne à Corbenic s'accompagne de chants, de prières, et d'un cortège rappelant la liturgie de la messe. Le déroulement du cérémoniel et la description du « saint vessel » varient assez peu d'un passage à l'autre. Pourquoi, alors, multiplier ces apparitions ? Il semble que chacun des trois chevaliers représente un cas différent : Bohort, le chevalier pur, appelé à la quête ; Gauvain, le chevalier réprouvé, trop «courtois » pour atteindre le degré de spiritualité nécessaire ; Lancelot, pour sa part, se situe dans l'entre-deux - ni pur, à cause de son amour adultère, ni tout à fait impur, à cause de la noblesse de cet amour. Le choix apparemment arbitraire des chevaliers admis en la présence du Graal obéirait donc à une logique supérieure, globale.

Dans le passage cité ci-dessus, Gauvain se merveille molt de l'aventure. Nous retrouvons les mêmes termes dans la bouche du roi :

${ }^{16}$ T. IV, LXXVIII, 51 ; T.VI, CVII, 30.

${ }^{17}$ T. IV, LXXXI, 12 et T.V, XCVIII, 24-25 ; 46-48. 
Et des aventures avez vos oïes de beles, puis que vos venistes, car aventure est ce merveilleuse, quant li Saint Graal nos donne chascun jor viande tel com nos demandons. ${ }^{18}$

Le terme de miracle, pourtant employé à un peu plus de dix reprises dans le Lancelot Propre, ne l'est pas pour désigner les scènes d'apparition du Graal, bien que l'intervention divine ne fasse pas de doute dans ces scènes, ne serait-ce que par le recueillement, les prières et les actions de grâce qui les accompagnent. Il semble que le terme de miracle soit employé dans le roman pour désigner l'action divine efficace, alors que le terme de merveille serait réservé au point de vue humain ${ }^{19}$ : or toutes ces scènes d'apparition du Graal sont décrites en focalisation interne, celle du héros spectateur qui s'étonne, admire et, submergé par le sublime, sait qu'il ne comprend pas. On rejoint ainsi la définition augustinienne du miracle, qui met l'accent sur l'effet produit sur le sujet :

Miraculum voco, quidquid arduum aut insolitum supra spem vel facultatem mirantis apparet. ${ }^{20}$

Les apparitions du Graal semblent ainsi défier les capacités interprétatives des spectateurs, qui ne dépassent guère le stade de l'émerveillement. En outre, la volonté qui préside aux apparitions du Graal prend soin de marquer les limites à ne pas franchir: Gauvain, qui regardait plus volontiers la porteuse du Graal que le Graal lui-même, n'a pas sa place au festin des justes, ne bénéficiera pas des pouvoirs nourriciers du Graal, et sera expulsé et humilié ; toute explication lui sera par la suite refusée ${ }^{21}$. Bohort lui-même, pour avoir vu le Graal découvert - préfiguration de sa participation à la quête - sera aveuglé et prié de ne pas espérer davantage :

\footnotetext{
${ }^{18}$ T.V, XCVIII, 26.

${ }^{19}$ Jean-René VALetTe, « Miracle et merveille dans les proses du Graal », dans Furent les merveilles pruvees, op. cit., p. 676 : « en d'autres termes, il revient à l'homme de se merveillier et à Dieu d'opérer des miracles. »

${ }^{20}$ Augustin, De utilitate credendi, XVI, 34, P.L., t. 42, col. 90.

${ }^{21}$ T. II, LXVI, 35.
} 
Au point que li prodom osta le samit de sus le Saint Graal, avint que leanz s'espandi si grant clarté qu'il fut avis à Boorz que an mi les eux l'eust feru .I. raiz de souleil : si an devint si esperduz que em perdi toute nuit la veue, si que il ne pot goute veoir. Lors oï une voiz qui li dist : «Boorz, ne vien plus avant, car tu n'ies pas dignes de veoir plus que veu as des secretes choses de ceanz. $\|^{22}$

Paradoxalement, ce Graal qui se donne à voir avec ostentation sait aussi se refuser et exclure les réprouvés. L'évidence de sa présence ne s'accompagne d'aucune explication rationnelle du spectacle imposé. Ses apparitions répétées ne dissipent guère son mystère, ou, pour reprendre le terme du texte ci-dessus, son secret.

Le merveilleux du Graal est si spectaculaire qu'il tend à éclipser le reste : le Lancelot Propre utilise cependant d'autres moyens moins évidents pour manifester la volonté divine, et ce bien plus régulièrement, dès le début du récit. Les emplois du terme de miracle dans le roman signalent la diversité de ces interventions :

Maintenant avint .I. miracle que cil de cest païs sevent bien, que l'eve de la fontainne qui devant estoit froide commança a boulir a granz ondes : einsi fu si chaude qu'il ot toutes les mains arses avant que il les am poïst avoir traites. Et quant il vit ceste merveille, si connut bien que Diex s'estoit correciez a lui, por ce qu'il avoit occis le prodome. ${ }^{23}$

Le terme de merveille se retrouve de façon récurrente dans l'ensemble du passage ; dans cet extrait-ci, la syntaxe semble suggérer que merveille est un strict équivalent de miracle, dont il constitue le rappel. Mais l'interprétation du phénomène d'ébullition de la fontaine comme un châtiment divin semble justifier néanmoins l'emploi de ce terme qui signale une intervention de la Providence. Nous retrouvons miracle étroitement associé au terme de merveille dans l'épisode de l'aventure du

${ }^{22} \mathrm{~T} . \mathrm{V}, \mathrm{XCVIII}, 46$.

${ }^{23} \mathrm{~T}$.V, XCIII, 15. C'est nous qui soulignons. 
blanc cerf et des lions :

Or sachiez [dit un saint ermite] que ce est une des graindres merveilles que onques veissiez ne ce n'est mie chose que vos puissiez achever ne vos ne home, fors seulement li bons chevaliers qui de bonté et de chevalerie passera touz les chevaliers terriens. Icil achevera l'aventure des lyons et dou cerf et fera savoir au monde par quel manniere li lion pristrent en garde le cerf: que bien saichiez vraiement que ce n'est mie anchantement ne obscure dyablie, ainz est miracle mervilleux qui avint jadis par la volenté Nostre Signor. ${ }^{24}$

L'épisode appelle plusieurs remarques. Une fois de plus, cette quête d'une explication à un phénomène étrange représente une simple aventure qualifiante destinée à caractériser Lancelot comme le meilleur chevalier du monde. Rien ne distingue à première vue cet épisode de nombreux autres du même type, où l'on retrouve de même les termes merveille et aventure. Mais, comme dans le cas précédent, miracle apparaît dès lors que l'interprétation met en jeu la volenté Nostre Signor, soit une intention divine qui s'opposera à une simple magie profane (anchantement) ou aux manœuvres diaboliques de la magie noire (dyablie). La sûreté de l'interprétation est ici garantie par la figure d'autorité en la matière que constitue le saint ermite.

En apparence, les hommes agissent librement, suivant leurs envies, leur conscience, parfois leur libido, en tout cas sans nulle intervention divine directe. Et pourtant, la Providence dispense constamment ses avertissements à travers divers intermédiaires humains qui peuvent être de saints ermites comme précédemment, souvent d'anciens chevaliers, des confesseurs, des abbesses, des demoiselles mystérieuses qui commentent l'action, sermonnent le roi ou les chevaliers, les conseillent ou leur annoncent des événements à venir. Tous reprennent le rôle laissé vacant par la disparition de Merlin. Ainsi Adragai le Brun fait-il prendre conscience à

${ }^{24}$ T.V, XCVI, 9. C'est nous qui soulignons. 
Arthur de la faute qu'il a commise envers Ban de Benoïc son vassal en ne le soutenant pas dans sa guerre contre Claudas :

« Rois Artus, fait il, Diex te saut com le plus preudom et le millor qui onques fust, se ne fust une seule chose. [...] Vous honerés et doutés et serves chex qui desloiaument vous guerroient et courent sus, et cheus oubliés qui vous ont loiaument servi et sans fauser, et les meteis arrière : si ont perdu terres et hounors et lor vies et sont en avanture de lor âmes perdre pour vostre service. Or vous ai devisee la chose por coi vous perdes a estre li plus preudons qui onques fust. »

Quant li rois l'entent, si en est moult hontex et par la sale en sont tout esbahi et un et autre et dient que onques mais n'avoient oï rendu si bien parler ne si hardiement devant si haut homme; et li plusor en ont le mangier laisié et a mervelles l'esgardent. [...] «ch'est la mort au roi Ban de Benoyc que vous ne venjastes onques, qui fu mors en la venue de vostre court. Et si est sa feme remese veve desiretee et s'est reubee d'un des plus biax enfans qui onques fust. C'est si laide chose et si vilaine a vostre oels qu'il est mervelle comment vous poés ne osés nul preudomme veoir en mi le vis. Et sachiés que nus pechiés ne vous destourbera tant a venir au desus de tout le monde comme chist. ${ }^{25}$

Cet homme, qui est un ancien chevalier d'Uter, parle à Arthur sur un ton d'autorité qui surprend tous les spectateurs, mais que l'on n'ose lui contester car il défend le droit. Certaines de ses paroles laissent à penser qu'il est investi d'une autorité qui peut être une inspiration divine notamment quand il annonce qu'aucun péché ne nuira davantage à Arthur que de n'avoir pas recueilli l'enfant de Ban. En effet, cet enfant n'est autre que Lancelot, et Arthur a perdu le lien vassalique qui l'attachait à lui, ce qui lui nuira beaucoup dans la Mort le roi Artu, où Lancelot se soulèvera contre lui. L'ermite se révèle donc visionnaire, et sa parole exprime une sagesse qui semble le dépasser, comme s'il était en quelque sorte le messager de la Providence. De même, lorsque l'ermite Segre expliquera à Gauvain le sens des événements qu'il a vécus à Corbenic : l'homme est capable d'expliquer une vision que le chevalier a eue d'un combat entre un serpent et un ${ }^{25}$ T. VII, XI a, 16-22. 
léopard, suivi d'une attaque de serpenteaux contre un grand serpent. Selon lui, cette vision prophétisait les démêlés à venir d'Arthur avec un chevalier étranger, puis ses propres vassaux. L'ermite n'explique pas d'où lui vient sa science $^{26}$. Un autre ermite annonce à Mordret qu'il tuera son père, et ce dernier, de fureur, le décapite ${ }^{27}$. Ce ne sont que quelques exemples parmi beaucoup d'autres. Aucun de ces personnages d'ermite n'aura de rôle durable auprès des héros, ils n'interviennent que ponctuellement et certains demeurent anonymes, n'étant que de simples instruments. Ces personnages secondaires ont une double action: ils tentent d'influencer les hommes, d'un côté, mais ils annoncent aussi des événements à venir déjà écrits, de l'autre, non pour avertir les hommes d'agir autrement, mais uniquement pour manifester la toute puissance divine. Après tout, à quoi bon annoncer à Mordret ce qu'il fera de toute façon ? Ainsi l'homme garde sa liberté d'action et c'est ce qui le rend responsable de ses actes, mais la sainte Providence démontre son omniscience de l'avenir, la supériorité de ses perspectives sur celles des hommes et, par conséquent, l'inévitable réussite de ses desseins.

Un autre procédé récurrent dans le roman est le procédé narratif des inscriptions de cimetière et des pierres tombales merveilleuses, emprunté à Chrétien de Troyes. On en trouve le premier exemple dans l'épisode de la Douloureuse Garde, premier exploit de Lancelot :

«Ceste lame n'iert ja levée par main d'omme ne par esfors, se par chelui non qui conquerra cest doleros castel et de che lui est li nons escris ci desous. » [...]

Lors le saisist a .I. mains par devers le plus gros, si l'a tant levee q'ele est plus haute que sa teste .I. pié. Et lors voit les lettres qui dient: "Chi gerra Lancelos del Lac, li fiex au roi Ban de Benoÿc. ${ }^{28}$

\footnotetext{
${ }^{26}$ T. II, LXVI, 37-38.

${ }^{27}$ T. V, XCVI, 26.

${ }^{28}$ T.VII, XXV a, 31-32.
} 
Ici c'est l'inscription qui apprend à Lancelot sa propre identité. Une autre inscription, sur le siège périlleux, rappelle à Brumant que c'est là qu'il mourra: il s'agissait à l'origine d'une prophétie de Merlin. Ces inscriptions sont mystérieuses car aucun renseignement n'est donné sur la façon dont elles sont apparues; le doute se maintient, du reste, sur leur nature : s'agissait-il d'une magie maléfique à la Douloureuse Garde, d'un avertissement divin ou de la magie de Merlin, au siège périlleux ? Le merveilleux des inscriptions maintient le doute sur leur origine comme sur l'interprétation à en donner.

Autre procédé très fréquemment employé pour faire intervenir la Providence, tout un appareil de songes prophétiques inquiétants ou d'avisions ${ }^{29}$, qui affectent en priorité Arthur ou Lancelot, est intégré régulièrement à la trame narrative. Par exemple, l'avision où Lancelot voit son aïeul le guider vers l'aventure de la Forêt Périlleuse. L'aïeul révèle à Lancelot l'origine de son nom, qu'il ignorait, et se présente lui-même comme un messager. Mais, s'il évoque avec insistance le rôle de la foi de la reine Hélène dans l'élection de Lancelot, il ne précise pas qui l'envoie.

Et quant il furent couchié par laienz, si avint a Lancelot une avision moult mervilleuse, car il li est avis que devant lui venoit .I. home viels et anciens qui l'apela par son droit non et dist : «Lanceloz, biaux niés, lieve sus et va en la Forest Perilleuse ou tu trouveras une aventure mervilleuse qui ne puet estre menée a chief fors par toi, et encor ne l'acheveroies tu pas, se ne fust la roine Heleinne, ta bonne mère, qui prie Nostre Signor por toi et nuit et jor. Et sez tu qui je sui, qui ça te fui venuz anuncier ceste chose? Je sui Lanceloz, qui fui rois de la Blanche Terre qui marchist au reaume de la Terre Foreinne, et sui tes aieux ; et por amor de moi et por honor t'apela li rois Bans mes filz Lancelot: ainsi as tu non am batesme Galaad. » ${ }^{30}$

29 "Les "avisions" sont une variété de rêves au cours desquels des personnages apparaissent au dormeur », Alexandre MICHA, Essais sur le cycle du Lancelot-Graal, Genève, Droz, 1987, p. 209.

${ }^{30}$ T.V, XCI, 29. 
Ce sera au lecteur d'identifier l'intervention divine dans cette surprenante apparition de l'ancêtre. Du reste, cette interprétation demeure discutable ; est-ce bien la Providence qui envoie l'aïeul donner cette mission à Lancelot? N'agit-il pas de son propre chef? Comme dans le cas des inscriptions et des propos des ermites, un acte de foi demeure nécessaire pour repérer la manifestation de la Providence.

Cette Providence est finalement très active dans le Lancelot Propre, non seulement dans l'Agravain, mais dans l'ensemble du roman. Néanmoins elle se dissimule, dispensant une sagesse à laquelle la plupart des personnages demeureront sourds. Sa manifestation n'a plus l'évidence d'une parole prophétique délivrée par un personnage bien identifié comme Merlin : sa parole se disperse dans la bouche de très nombreux personnages secondaires dont une bonne partie reste anonyme, se retrouve gravée sur la pierre ou nimbée du mystère du songe ou de la vision merveilleuse allégorique. Une partie de ce discours est révélée : ainsi, les songes sont toujours interprétés. Mais les intentions de cette Providence ne sont pas toujours claires : elle souhaite quelquefois influencer, et le reste du temps semble se contenter d'annoncer l'inéluctable, peut-être en guise de premier châtiment. Avec le recul, le lecteur prend conscience que la Providence a la connaissance exacte de la suite des événements, ce qui amène le narrateur à se livrer à de nombreuses prolepses. La Providence est ainsi le réel conducteur de l'Histoire, le seul véritable auteur du récit. Les héros apparaissent n'être finalement que de simples outils entre ses mains, alors que son dessein ultime semble n'être rien de moins que de réactualiser, à travers la quête du Graal, le sacrifice christique. 